\title{
The sandman's secrets
}

\author{
Neuroscientists at last have a molecular handle \\ on how the brain controls our daily cycle of sleep \\ and wakefulness. This is opening a rich avenue of \\ study that could lead to new therapies for sleep \\ disorders, says Marina Chicurel.
}

et sleeping dogs lie, warns the old proverb. But Emmanuel Mignot has helped to open up an exciting area of molecular neuroscience by ignoring this advice. In 1989, Mignot and his colleagues at Stanford University School of Medicine in California began to study a group of Dobermann pinschers afflicted by narcolepsy - a disorder marked by uncontrollable urges to sleep and sudden bouts of muscle weakness. They hoped that the dogs would provide a suitable animal model in which to uncover the causes of this distressing human disease.

More than a decade later, that hope is being realized. But, more significantly, Mignot's project has also converged with several other lines of research to suggest that a pair of neuropeptides called the hypocretins/orexins are central players in controlling the body's cycle of sleep and wakefulness. As such, the peptides should help fill an important knowledge gap.

Biologists have made great strides in understanding the molecular components of the internal clocks that control our daily, or circadian, biological rhythms. But they have struggled to reveal how these clocks influence our sleep patterns. "The link between circadian rhythms and sleep is one of the great unsolved problems," says Allan Hobson, a leading sleep researcher at Harvard Medical School in Boston. "These neuropeptides are the first specific candidates."

With millions of people suffering from sleep disorders, the pharmaceuticals industry is interested in the hypocretins/orexins - better sleeping pills, drugs to combat drowsiness and treatments for conditions

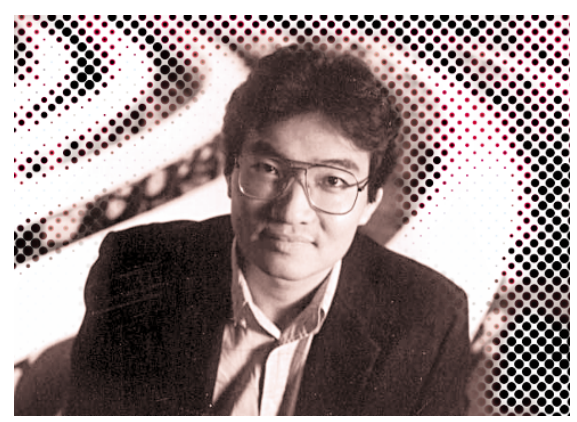

Yanagisawa: infrared snooping on knockout mice offered clues to the causes of narcolepsy. such as narcolepsy could emerge from research on the neuropeptides.

The peptides should also shed light on other elements of the body's 'housekeeping' processes - such as the control of appetite and blood pressure. "It's an amazing culmination of a series of leads that have come together over a relatively short period of time," says Thomas Kilduff, a neuroscientist at SRI International, a non-profit research corporation in Menlo Park, California.

\section{Clocking on}

One of the earliest leads emerged from Gregor Sutcliffe's lab at the Scripps Research Institute in La Jolla, California. In 1994, he started looking for molecules that regulate basic body functions such as eating, sleeping and reproduction. Neuroscientists had long known that a region in the brain called the hypothalamus controlled most of these processes by producing specific peptides to transmit signals within the brain and to other organs. But the number of known neuropeptides was small compared with the range of activities governed by the hypothalamus. "We argued there must be a lot of hardware left to discover," says Sutcliffe.

So Sutcliffe and his colleagues searched for genes that were expressed only in the hypothalamus. Using messenger RNA (mRNA) extracted from rat hypothalamus tissue, the researchers generated a library of single-stranded complementary DNA (cDNA). To separate out genes expressed in the brain in general from those specific to the hypothalamus, the workers mixed all the fragments with mRNA from two other brain regions. Many of the cDNAs paired up with these mRNAs, forming double-stranded molecules. But 38 of the fragments could find no partner and remained single-stranded.

One in particular, dubbed 'clone 35', attracted the researchers' attention because its mRNA was found exclusively within a cluster of cells in the lateral hypothalamus ${ }^{1}$. Because such cell clusters tend to regulate specific functions, clone 35 seemed to fit the bill for the type of regulatory hardware the researchers were looking for. "As soon as we saw that clone," recalls Kilduff, a member of the research team, "we knew we had struck gold." Sutcliffe and his colleagues predicted

$\approx \odot 2000$ Macmillan Magazines Ltd
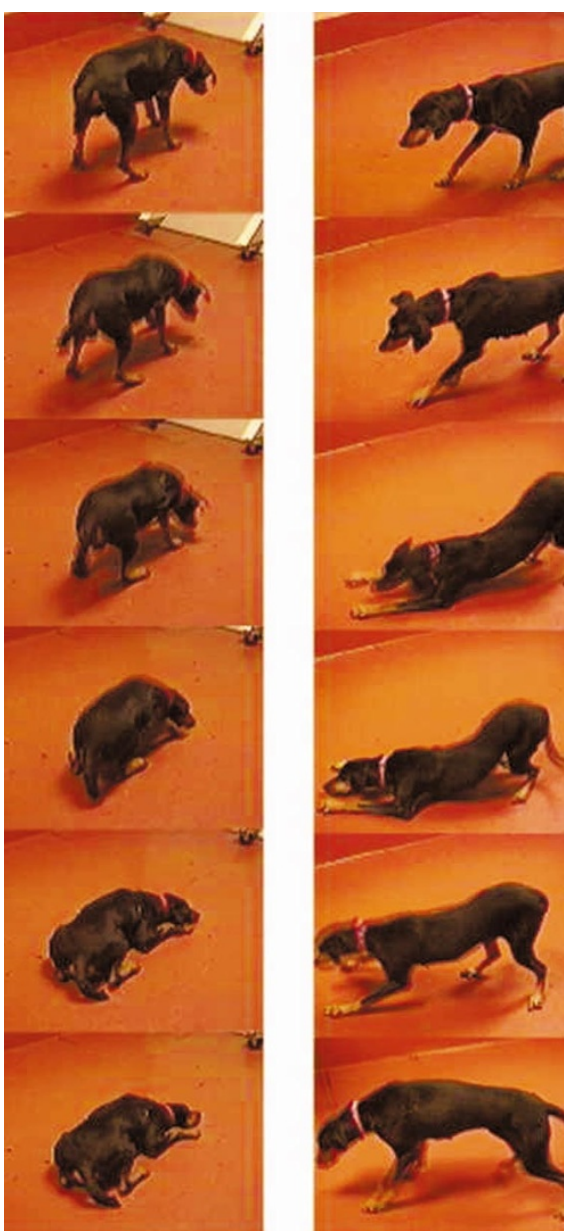

Dog tired: studies of narcoleptic Dobermann pinschers have linked the control of sleep to two peptides that are thought to regulate appetite.

the structure of two peptides encoded by clone 35 and named them hypocretins, because they were produced in the hypothalamus and resembled the incretin neuropeptides, which help regulate gut function.

Meanwhile, at the University of Texas Southwestern Medical Center at Dallas, Masashi Yanagisawa and his colleagues were rummaging through a mass of 'orphan' receptor molecules. Found on the surface of cells, these molecules transmit a signal to the cell's interior when they bind to neuropeptides or other transmitter molecules. Genomics studies had identified over 100 human DNA sequences as coding for surface receptors coupled to $G$ proteins, which are important in cell signal transduction. But most of these receptors had no known partner. Yanagisawa reasoned that if he could find some of the transmitters that bound to them, he would gain insights into brain function - with possible medical applications. "Sixty per cent of clinically used drugs target one or more of the G protein-coupled receptors," he notes.

Sifting through brain extracts, Yanagisawa's team discovered two peptides, derived from a single precursor protein, that stimulated two closely related orphan receptors ${ }^{2}$. Again, the peptides were produced in the 

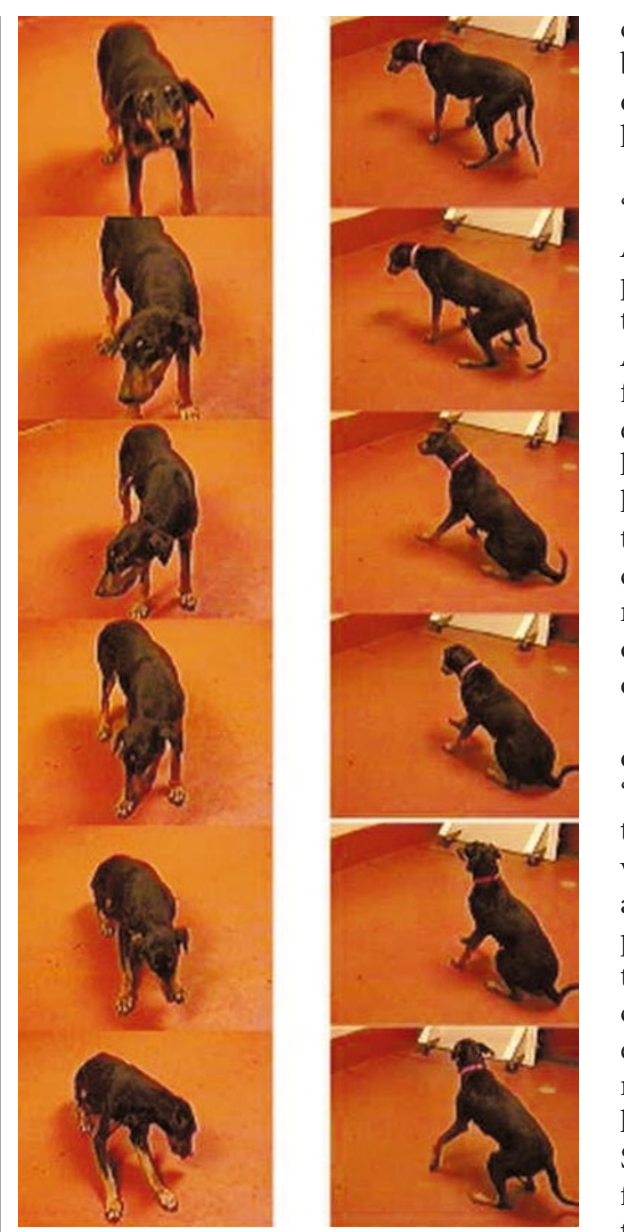
brain, but the other encoded one of the two orphan receptors studied by Yanagisawa, the hypocretin/orexin 'type 2' receptor.

"It was a good candidate," says Mignot. "But at the same time we weren't so sure." After years of painstaking effort, and one previous false alarm ${ }^{3}$, Mignot was reluctant to focus all of his efforts on a single receptor. Also, he could not quite believe that the gene for which he had long been searching encoded a receptor for neuropeptides that a former labmate had just helped discover - Kilduff had worked with Mignot at Stanford before teaming up with Sutcliffe. "It was such a coincidence, it was impossible," says Mignot, who now reckons that he could have ended his quest six months earlier, had he ignored his disbelief and followed the lead.

By the time Mignot had dispelled his doubts, Yanagisawa's group had created 'knockout' mice in which the genes encoding the two neuropeptides were disabled. Convinced that the peptides primarily regulated appetite, Yanagisawa and his colleagues probed their mice for feeding abnormalities, taking the unusual step of using an infrared camera to monitor the animals' nocturnal eating habits. This night-time snooping revealed that the mice would suddenly collapse, often while walking or grooming. Suspecting that the animals were suffering from seizures, the researchers used electrodes to monitor the rodents' brainwaves. They found that, far from experiencing seizures, the mice were falling asleep. "These mice fulfilled all the objective criteria of human narcolepsy," says Yanagisawa. In August last year, only two weeks after Mignot published his findings on narcoleptic $\operatorname{dogs}^{4}$, Yanagisawa reported his knockout-mouse study ${ }^{5}$.

Since then, the link between the neuropeptides and human narcolepsy has strengthened. First, Mignot and his colthe Greek word orexis, meaning appetite.

Sutcliffe and Yanagisawa only realized the overlap between their work after they had published their papers, just six weeks apart. Sutcliffe's hypocretin mRNA, it turned out, encoded the two orexins. But it took another coincidence - this time involving a convergence between Yanagisawa's work and Mignot's narcoleptic dog project — to make the connection with sleep.

\section{Unlikely bedfellows}

Mignot had been struggling to identify genes associated with narcolepsy for the best part of a decade. But in 1997, the pace picked up after his team and other collaborators built a library containing large DNA fragments from the genome of a dog that was heterozygous for a narcolepsy gene - carrying one copy of the normal gene and one of the mutated, disease-causing version. In less than two years, the team narrowed the search for the gene to a genomic region containing two leagues measured the levels of one of the neuropeptides, hypocretin 1/orexin-A, in the cerebrospinal fluid of nine narcoleptic patients. Seven had levels below the detection limit ${ }^{6}$. And just last month, Mignot's group and a team led by Jerome Siegel at the University of California at Los Angeles published separate papers revealing that post-mortem brains of human narcoleptics contain almost no hypocretins/orexins $s^{7,8}$. But unlike Mignot's dogs, human narcoleptics only very rarely seem to have mutated hypocretin/orexin genes ${ }^{7}$. Instead, it seems that they have lost most, if not all, of the cells that produce the neuropeptides. Siegel found scar tissue in the area normally occupied by the hypocretin/orexin-producing cells $^{8}$, consistent with the idea that narcolepsy might be an autoimmune disorder ${ }^{9,10}$.

\section{Dreams of drugs}

Neuroanatomical studies have reinforced the idea that the neuropeptides help control the sleep-wake cycle" ${ }^{11}$. All the important structures in sleep regulation receive a hypocretin/orexin input," says Christelle Peyron, who recently moved from Stanford to the Geneva University Hospitals, and who first described the distribution of the hypocretins/orexins in the brain. This has led researchers to speculate that research on the peptides could result in new drugs to treat a variety of sleep disorders. Drugs that mimic the neuropeptides, for instance, might do for narcoleptics what insulin does for diabetics. And if the basic role of the hypocretins/orexins is to maintain wakefulness or act as a 'gatekeeper' of sleep, they might also combat normal sleepiness such as that experienced by night-shift workers. Conversely, molecules that block the neuropeptides' effects might be used to develop improved sleeping pills.

But many hurdles lie ahead." The pharmacology of the peptides is in its infancy," says Neil Upton of SmithKline Beecham in Harlow, Essex, which holds commercial rights to aspects of Yanagisawa's work. Treating narcolepsy using hypocretin/orexin mimics is the most immediate prospect. But Upton and his colleagues have already generated a small molecule that crosses the blood-brain barrier and binds to the type 1 hypocretin/orexin receptor, preventing it from being stimulated by the neuropeptides. Hypocretins/orexins increase arousal when injected into the brains of rats ${ }^{12}$, but SmithKline Beecham's molecule seems to reverse this effect. The team has yet to assess whether the molecule will promote sleep in normal rats that have not received hypocretin/orexin injections, and need to design more stable molecules that will persist in the brain for several hours.

Another problem is that tinkering with the hypocretin/orexin system could do more than just influence sleep. SmithKline Beecham's molecule reduces appetite - an effect for 
